\title{
STATISTICAL PROPERTIES OF AURORAL KILOMETER RADIATION: BASED ON ERG (ARASE) SATELLITE DATA
}

\section{V.I. Kolpak}

Space Research Institute of RAS,

Moscow, Russia, lera.kolpak@yandex.ru

National Research University "Higher School of Economics",

Moscow, Russia, lera.kolpak@yandex.ru

\section{M.M. Mogilevsky}

Space Research Institute of RAS,

Moscow, Russia, mogilevsky2012@gmail.com

\section{D.V. Chugunin}

Space Research Institute of RAS,

Moscow,Russia,dimokch@mail.ru

\section{A.A. Chernyshov}

Space Research Institute of RAS,

Moscow, Russia, achernyshov@iki.rssi.ru

\section{I.L. Moiseenko}

Space Research Institute of RAS,

Moscow, Russia, moiseenko.irine@gmail.com

\section{A. Kumamoto}

Tohoku University,

Sendai, Japan, kumamoto@stpp.gp.tohoku.ac.jp

\author{
F. Tsuchiya \\ Tohoku University, \\ Sendai, Japan, tsuchiya@pparc.gp.tohoku.ac.jp
}

Y. Kasahara

Kanazawa University,

Kanazawa, Japan, kasahara@is.t.kanazawa-u.ac.jp

M. Shoji

Nagoya University,

Nagoya, Japan, masafumi.shoji@nagoya-u.jp

Y. Miyoshi

Nagoya University,

Nagoya,Japan,miyoshi@isee.nagoya-u.ac.jp

\section{Shinohara}

Institute of Space and Astronautical Science, Japan Aerospace Exploration Agency,

Tokyo, Japan, iku@stp.isas.jaxa.jp

\begin{abstract}
In this work, we have studied the signals of auroral kilometer radiation (AKR) from sources in the auroral regions of the Northern and Southern hemispheres simultaneously recorded by one satellite. We have carried out a detailed statistical analysis of more than 20 months of continuous AKR measurements made by the ERG satellite (also known as Arase). This made it possible to confirm the previously obtained results on the location of AKR sources and seasonal changes in the radiation intensity. Open questions about the pro-
\end{abstract}

cesses in the AKR source can be solved using data on the radiation pattern under various geomagnetic conditions. To answer these questions, we have estimated the cone angle of directional diagrams in the dusk and dawn sectors of Earth's magnetosphere.

Keywords: auroral kilometer radiation, magnetosphere, auroral activity, satellite measurements.

\section{INTRODUCTION}

Auroral kilometer radiation (AKR) is powerful natural electromagnetic radiation in the $30-800 \mathrm{kHz}$ frequency range (maximum radiation is observed from 100 to $300 \mathrm{kHz}$ ), which is generated in near-Earth plasma and propagates from Earth. AKR was first recorded by the satellite Elektron-2 in 1965 [Benediktov et al., 1965]. Already the first studies on AKR identified important properties of this radiation - frequency range, radiation polarization, radiation intensity dependence on geomagnetic activity, etc. [Gurnett, 1974; Kurth et al., 1975; Benson, Calvert, 1979]. Early studies on the AKR nature have found that sources of this radiation are situated in the auroral region, are linked to discrete auroras, and are located in the dusk and night sectors of the magnetosphere at invariant latitudes of $\sim 70^{\circ}$ at altitudes $(2 \div 10) 10^{3} \mathrm{~km}$ [Kurth et al., 1975; Benson, Calvert, 1979]. The mechanism of AKR generation remained unknown for more than a decade after the discovery of the radiation until publication of the article [Wu, Lee, 1979], in which the authors proposed using the cyclotron maser instability mechanism to interpret AKR. Such instability develops in the auroral magnetosphere in regions with low plasma density, where the electron plasma frequency is lower than the local electron gyrofrequency $\omega_{\mathrm{pe}}=\left(4 \pi \mathrm{e}^{2} N / m\right)^{1 / 2}<\omega_{\mathrm{Be}}=e B /(m c)$. The source of the radiation energy is superthermal electrons (several $\mathrm{keV}$ ), which are injected from the magnetotail into its inner regions during geomagnetic disturbances. AKR is generated at a local electron gyrofrequency, and in its source it is transverse to the magnetic field. Subsequent studies [Louarn, Le Quéau, 1996; Burinskaja, Rosh, 2007] have shown that the radiation source includes a waveguide that "stretches" radiation along magnetic field lines, and walls of this waveguide may be not only the background plasma density gradient but also the superthermal particle flux boundary [Burinskaja, Rosh, 2007].

Chugunin et al. [2020] have investigated a regular change in AKR fluctuation wavelet spectra along a magnetic field line provided that at a high altitude the 
fluctuation spectrum is power-mode and at a low altitude the fluctuation distribution becomes uniform for all characteristic times, which indicates complex nonlinear processes in the AKR source.

AKR is generated due to the interaction between plasma flows from the Sun (star) and planetary magnetic fields. The radiation similar in nature (but not in frequency) was detected from Jupiter, Saturn, Uranus, and Jupiter satellite Io because radiation of this type is characteristic of space bodies having a magnetic field [Zarka, 1998].

Nowadays, the basic properties of AKR are well known, which allows us to use this radiation for diagnosing processes in Earth's magnetosphere. The open questions related to processes in the AKR source can be addressed using data on radiation pattern under different geomagnetic conditions. This paper deals with these problems.

\section{ERG SATELLITE AND ITS ORBIT}

The Japanese satellite ERG (Exploration of Energization and Radiation in Geospace) was launched on December 20, 2016; after reaching orbit, it was renamed Arase (after the river near the launching point) [Miyoshi et al., 2018a, b] and has been successfully operating since then. This spacecraft is $\sim 350 \mathrm{~kg}$ in weight, has an orbit with apogee of $\sim 32000 \mathrm{~km}$, a perigee of $\sim 400 \mathrm{~km}$, an inclination of $31^{\circ}$, and an orbital period of $\sim 570 \mathrm{~min}$. The main objective of this space mission is to study physical processes in radiation belts. To fulfill it, the satellite's orbit has been chosen such that the apogee evolves with a period of $\sim 14$ months both in the XY plane and along the $\mathrm{Z}$ axis.

AKR measurements by the ERG satellite are of great interest because they enable the direct comparison of the radiation from sources located in the Northern and Southern hemispheres. Although studying AKR is not the primary objective of the satellite measurement program, measurement capabilities of onboard equipment facilitate this research. To study AKR, we have measured the electric-field component in the PWE/HFA experiment [Kumamoto et al., 2018], aimed at measuring the constant electric field and plasma waves in the frequency range from the constant field to $10 \mathrm{MHz}$ for the electric field and from a few hertz to $100 \mathrm{kHz}$ for the magnetic field [Kasahara et al., 2018].

For Earth's inner magnetosphere in a first approximation, we may assume that the plasma density varies in proportion to the absolute value of the magnetic field. Simulation results show that at frequencies above 100 $\mathrm{kHz}$ the plasmaspheric effect on radiation propagation is insignificant and we can adopt a simple geometrical scheme [Xiao et al., 2016].

It is known that at the output of the source AKR generally has electron (right) polarization in the Northern Hemisphere. The ERG satellite can detect radiation with opposite polarization directions from northern and southern sources, thus providing information on the AKR source location. In the polarization spectrogram (Figure 1), the blue color corresponds to northern sources;

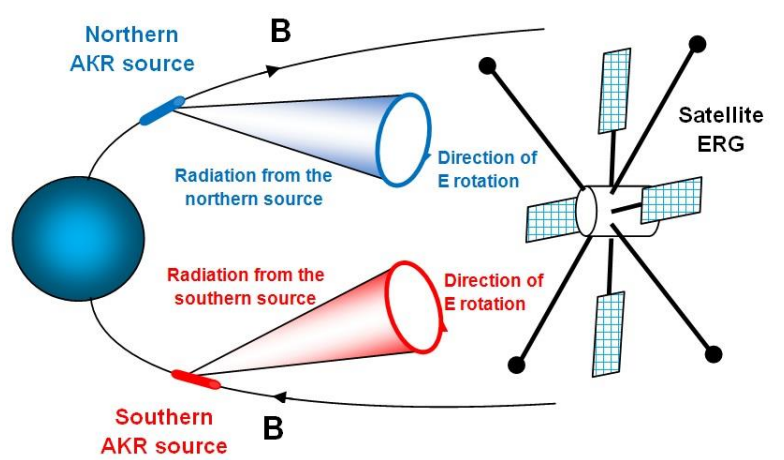

Figure 1. A diagram explaining the difference in the parameter $\left(E_{r}^{2}-E_{1}^{2}\right) /\left(E_{r}^{2}+E_{1}^{2}\right)$ as a function of the source location. $\mathrm{B}_{0}$ is the constant geomagnetic field.

red, to southern ones. Analyzing AKR requires not the relative polarization parameter as such but the zero crossing of this parameter, which indicates the dominant position of the power flux. Joint consideration of the radiation power density spectrum and the polarization spectrum allows us to analyze concurrent phenomena occurring in northern and southern auroral regions.

\section{STATISTICAL PROCESSING OF AKR MEASUREMENTS BY THE ERG SATELLITE AND ANALYSIS OF THE RESULTS}

In this paper, we have used results of preprocessing of electromagnetic field measurements made during the PWE experiment in a frequency range from a few kilohertz to $15 \mathrm{MHz}$ [Kasaba et al, 2017; Kasahara et al., 2018].

The AKR measurements were selected using the standard spectral signal processing. The ERG satellite data is available on the project website [https://ergsc.isee.nagoya-u.ac.jp]. Electric components were measured nearly continuously, which after statistical processing provided a uniform picture of radiation intensity distribution for long periods from March

2017 to December 2018. During that time, due to evolution of the satellite's orbit the $z$ component of apogee completed a full cycle from the Northern Hemisphere to the Southern Hemisphere (Figure 2, a). The magnetic local time MLT of the apogee is shown in panel $b$. In the selected interval, the apogee MLT variations exceed $24 \mathrm{hrs}$, thus giving us a full picture of AKR intensity distribution, i.e. the satellite apogee has made a complete rotation in the ecliptic plane.

The electric component measurements presented on the website [https://ergsc.isee.nagoya-u.ac.jp/cef/test/ erg_test.cgi?site] were divided into two-hour intervals with the radiation intensity analyzed in a frequency range $100-800 \mathrm{kHz}$ and the typical AKR value found. The resulting series was used for analyzing the integral intensity, and at the first stage we averaged over 30 days (more than the solar rotation period) to increase the accuracy of values and to minimize the influence of geomagnetic activity. The resulting AKR 

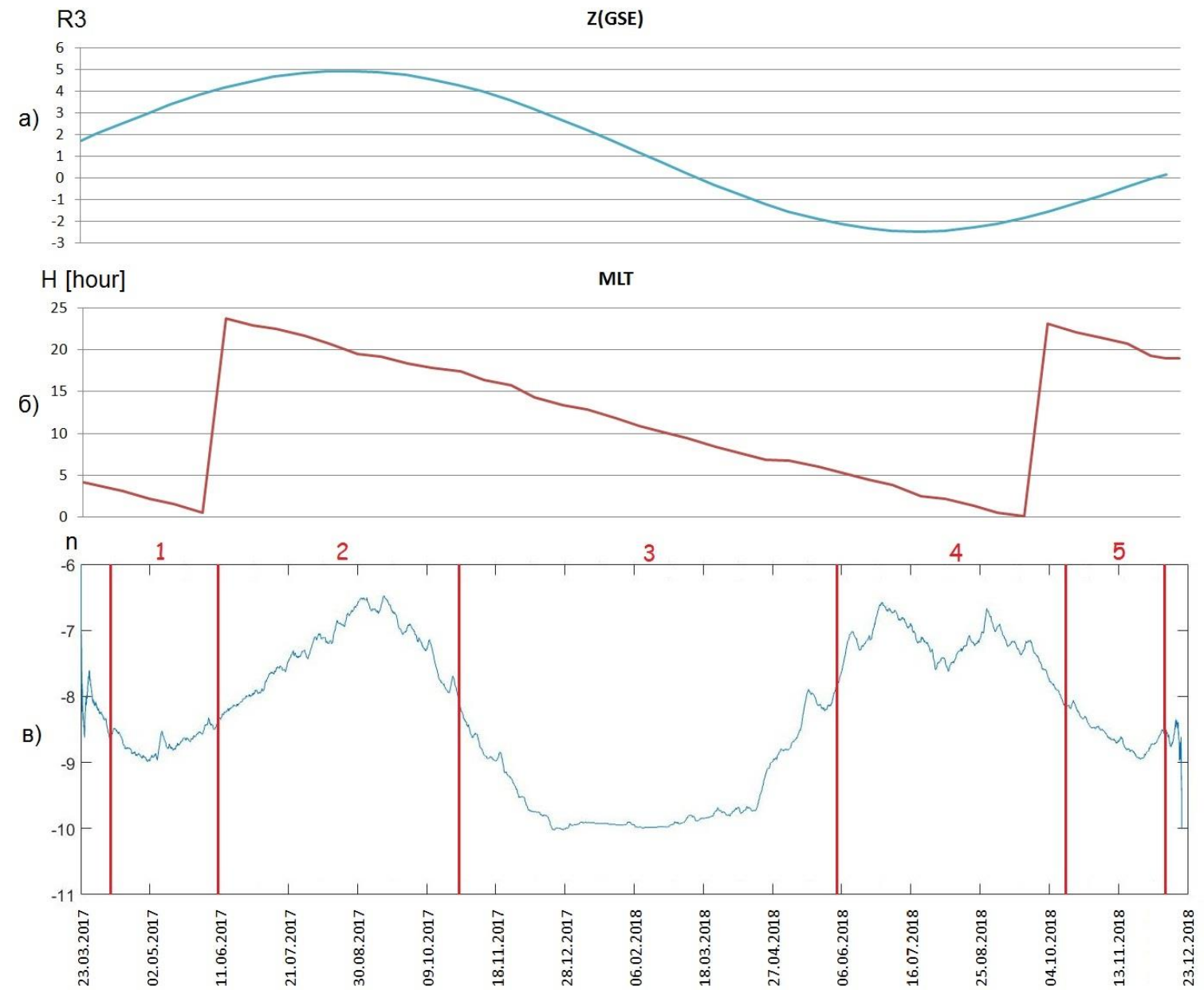

Figure 2. Results of statistical processing of AKR measurements from March 2017 to December 2018

integral intensity curve is shown in Figure 2, $c$. There are several characteristic intervals depending on observation conditions (red):

- interval 1, March 23, 2017 - June 01, 2017 the apogee of the satellite orbit was in the dawn sector of the magnetosphere near the ecliptic plane in the Northern Hemisphere;

- interval 2, June 01, 2017 - October 23, 2017 the orbit was mainly in the Northern Hemisphere in the midnight and dusk sectors of the magnetosphere;

- interval 3, October 23, 2017 - June 01, 2018 most of the orbit was in the dayside magnetosphere;

- interval 4, June 01, 2018 - October 10, 2018 the apogee of the orbit was in the Southern Hemisphere in the region favorable for generating AKR - in the dawn and night sectors;

- interval 5, October 10, 2018 - December 23, 2018 - similar to interval 1, but for the Southern Hemisphere.

The lowest AKR integral intensity is in interval 3. As has been shown in a number of papers such as [Benson, Calvert, 1979], AKR sources are located in the dusk, night, and dawn sectors of the magnetosphere between 18 and 06 MLT (Figure 3). Nonetheless, in Figure 2 we can see the so-called "inflowing" of AKR into the daytime sector: radiation is recorded outside of the location of sources - in the dusk (15-18 MLT) and dawn (6-8 MLT) sectors. This is due to the finite size of the radiation pattern: in the dusk sector the «inflowing» at 3 MLT corresponds to an opening angle of the cone of $\sim \pm 45^{\circ}\left(3^{\circ} \times 15^{\circ}\right)$, and in the dawn sector the opening is $\sim \pm 30^{\circ}$. This difference may be due to different conditions in the source. Perhaps because of the gradient drift the superthermal electron density in the dawn sector is higher, thus causing the waveguide length to increase and the radiation cone to narrow. The radiation cone opening values are generally consistent with the results obtained by another method [Mogilevsky et al., 2007; Benson, 1982]. However, the full understanding of the difference between radiation cone openings in the dusk and dawn sectors requires further research.

In interval 2 (Figure 2), the satellite apogee was shifted toward the Northern Hemisphere $(z>0)$, and therefore the radiation was generally observed from northern sources. Such a conclusion can be drawn from the analysis of the ratio between left and right polarization amplitudes. The maximum integral power of AKR is observed at 20 MLT, and then its intensity begins to gradually decrease (Figure 2). This decrease might be associated with a decrease in $z_{\mathrm{GSE}}$, which leads to a deterioration in observation conditions of AKR from sources in the Northern Hemisphere. Figure 4 shows the results of spectral analysis of AKR recorded on June 6, 2017 as typical measurements 

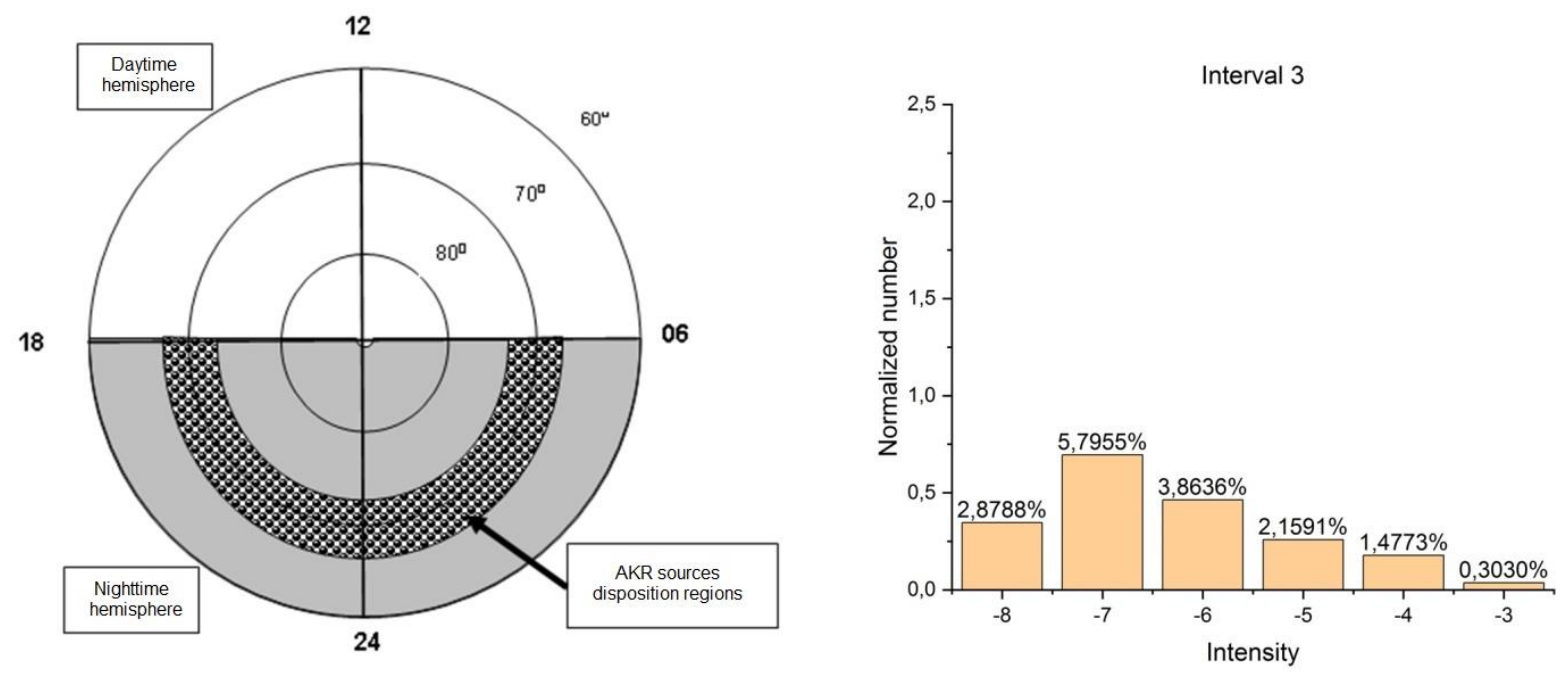

Figure 3. Universally accepted location of AKR sources in coordinates $\varphi_{\mathrm{m}}$ (geomagnetic latitude) and MLT (left). Schematic AKR spectrum in interval 3 (right). Intensity - powers of ten, $\mathrm{mV}^{2} /\left(\mathrm{m}^{2} \mathrm{~Hz}\right)$. Above the normalized number of two-hour intervals is the per cent of observed radiation of the total number of measurements
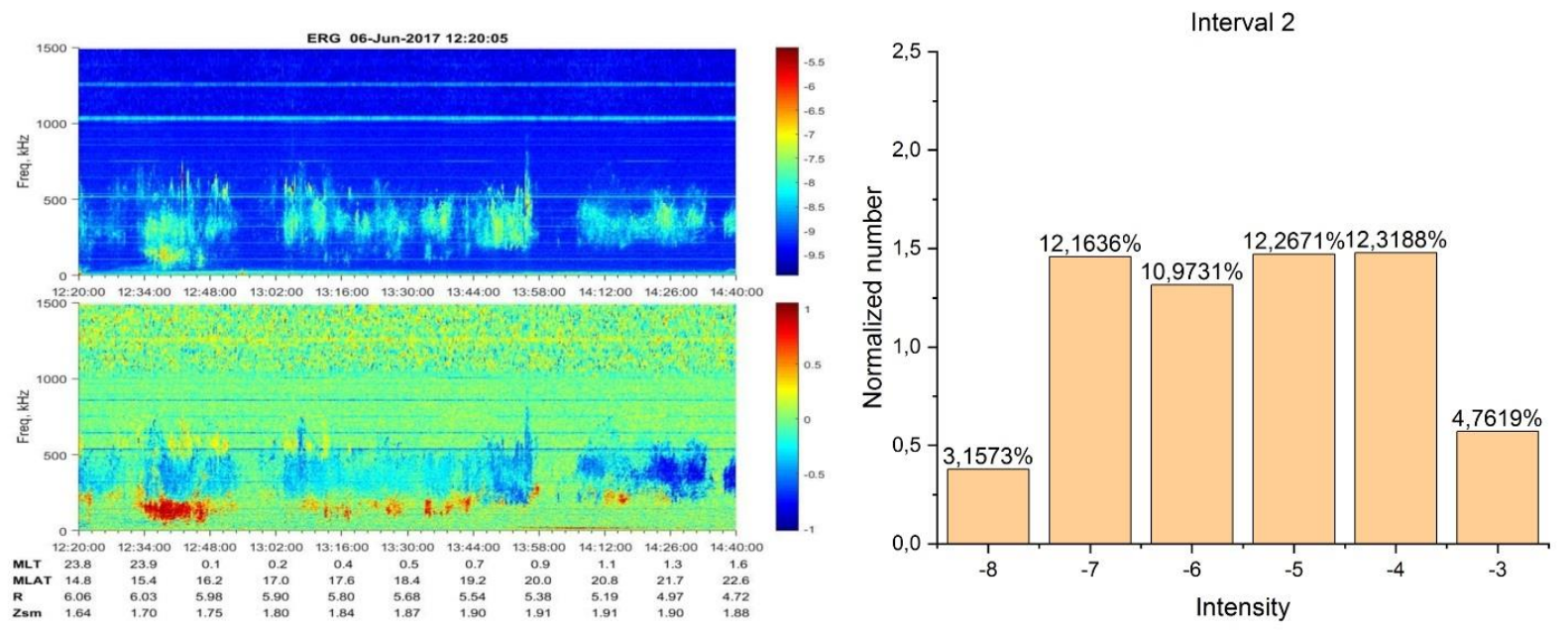

Figure 4. Spectral analysis of AKR recorded on June 6, 2017 (left). A dynamic spectrogram of power spectrum (top), a spectrogram of polarization parameters $\left(E_{r}^{2}-E_{1}^{2}\right) /\left(E_{r}^{2}+E_{1}^{2}\right)$ (bottom). A schematic power density spectrum of AKR recorded across interval 2 (as in Figure 3) (right).

of radiation in interval 2, as well as a schematic spectrogram of the AKR intensity across interval 2. When comparing the schematic spectrograms in Figures 3 and 4, we find the following differences:

- intensities (number of intervals) of all amplitudes in interval 2 is much higher than in interval 3;

- The schematic spectrum in interval 3 is incident (the minimum value is invalid as being noisy) and in interval 2 the spectrum is almost flat.

The difference between the intensities is attributed to the lack of radiation sources on the dayside, and the difference in nature between schematic spectra is likely to arise from the inflowing of radiation into this region and from the absence of sources as such.

In AKR spectrograms (Figure 4, left), we can identify radiation of three types. The lowest-frequency radiation from 100 to $300 \mathrm{kHz}$ has a maximum intensity at $\sim 12: 40$, its source is in the Southern Hemisphere, and duration is longer than $\sim 2 \mathrm{hrs}$. The radiation is marked with red in the bottom polarization spectrogram. Radiation of another type in the frequency band from 300 to $600 \mathrm{kHz}$ (with separate bursts up to $\sim 800 \mathrm{kHz}$ ) has an intensity comparable with the southern radiation, is recorded throughout the observation period, and has a source in the Northern Hemisphere (dark blue and light blue in the polarization spectrogram). The transition from dark blue to light blue and back may be attributed to the simultaneous recording of the radiation of the two types from northern and southern sources in the same frequency bands. In this case, at constant amplitude of radiation, polarization coefficients decrease, which is seen in the bottom spectrogram. From 12:20 to 13:16 there is still higher frequency radiation $500-700 \mathrm{kHz}$. Polarization of this radiation points to a source located in the south. This is likely to be the second or third AKR harmonic from the same southern sources [Benson, 1982]. Note also that frequency separation of spaced sources may take place: 
radiation from remote (more poleward) sources is recorded in the spectrogram at low frequencies; and radiation from nearby (more equatorward) sources, at higher frequencies.

In interval 4 (Figure 2), position of the satellite's apogee was shifted toward the Southern Hemisphere $(z<0)$, and the satellite recorded radiation mostly from southern sources. In the Southern Hemisphere, it was local winter at that time, and therefore the mean intensity is much higher than in the previous interval (greater than the maxima).

Similarly to interval 2 , the top spectrogram depicts an AKR region typical for this interval. The bottom spectrogram shows from which hemisphere this radiation came. In the spectrogram, red color prevails, which implies the radiation came from southern sources.

In Figure 5, spectrograms show radiation at frequencies above $800-1000 \mathrm{kHz}$, which are harmonics of the primary radiation from the southern source, but there are no such harmonics from the northern source. This situation also occurs at 11:46 when radiation from the northern source prevails. This indicates the spatial filtering we have discussed above. The schematic power density spectrum of the AKR recorded in interval 4 is close in power and intensity to measurements in interval 2 - the intensity distribution is nearly flat.

At 11:00-11:23 in the frequency band $1.3-1.5 \mathrm{MHz}$ there is a continuum radiation - synchrotron radiation of radiation belt electrons.

In the schematic power spectra in Figures 4, 5, each subsequent value differs from the previous one by an order of magnitude, and therefore the highest power corresponds to the right column $-10^{-3} \mathrm{mV}^{2} /\left(\mathrm{m}^{2} \mathrm{~Hz}\right)$. Comparison of the values in Figures 4, 5 shows that in interval 4 the mean radiation intensity is almost twice as high as that in interval 2. This is due to the seasonal dependence of the AKR radiation intensity. Measurements in interval 2 were carried out in the Northern Hemisphere in summer $(z>0)$, while in interval 4 in the Southern Hemisphere it was local winter $(z<0)$. The papers [Kasaba et al., 1997; Mogilevsky et al., 2005] have shown that during summer heated ionospheric plasma flows emerge from the auroral ionosphere into the magnetosphere and suppress AKR radiation generation at low altitudes. As such, the AKR spectrum becomes narrower, thereby decreasing the integral radiation intensity.

When comparing the AKR mean intensity in intervals 2 and 4 , we may note the presence of two intensity peaks in interval 4. The intensity increase between 08 and 05 MLT is quite understandable and is linked to the size of the cone of the AKR source radiation (see above). But the subsequent decrease in the mean intensity and the minimum at $\sim 2.5$ MLT have no explanation yet. The presence of this minimum seems especially strange since the apogee of the satellite orbit at that time was at a minimum value of $z$, so there were the best conditions for detecting AKR from southern sources. We plan to analyze this feature in detail in follow-up studies.

\section{CONCLUSION}

We have first investigated AKR signals from sources in auroral regions of the Northern and Southern hemispheres simultaneously recorded by one satellite (ERG). Statistical processing of a large AKR dataset (more than 20 months of continuous measurements) gave the following results.

- We have examined AKR near the equatorial region and analyzed radiation properties.

- We have confirmed the earlier results on the location of AKR sources, in particular on the possibility of appearance of several sources in the auroral region and seasonal variations in radiation intensity — intensity decrease in summer and increase in winter.

- We have estimated the AKR radiation pattern cone: in the dusk sector of the magnetosphere the opening angle of the cone is $\pm 45^{\circ}$; in the dawn sector, $\pm 30^{\circ}$.

ERG (Arase) satellite data as well as specification and characteristics of scientific instruments were received from the ERG Research Center, led by the Institute of Space and Astronautical Science of the Japan
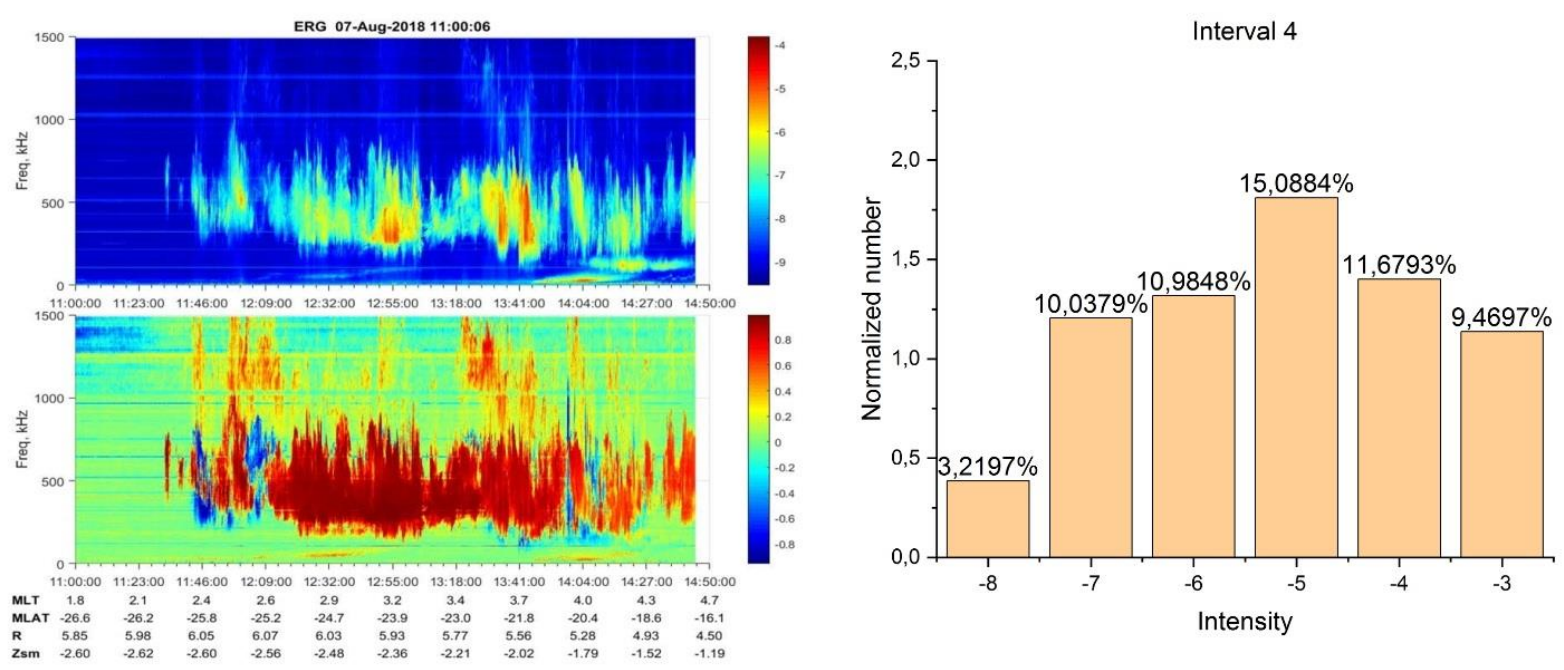

Figure 5. Spectral analysis of AKR detected on August 7, 2018 (left). A dynamic spectrogram of power density spectrum (top), a spectrogram of polarization parameters (bottom). A schematic power density spectrum of AKR recorded across interval 2 (right) 
Aerospace Exploration Agency and ISEE/Nagoya University [https://ergsc.isee.nagoya-u.ac.jp, Miyoshi et al., 2018b].

The study analyzes PWE HFA-L2 v01.01. data [Kumamoto et al., 2018].

This work was funded by RFBR (project No. 18-2921037).

\section{REFERENCES}

Benediktov E.A., Getmancev G.G., Mitjakov N.A., Rapoport V.A., Sazonov Ju.A., Tarasov A.F. Results of measuring the intensity of radio emission at 725 and $1525 \mathrm{kHz}$ frequencies using the equipment installed on "ELECTRON-2" satellite. Issledovanija kosmicheskogo prostranstva: [Space exploration]. Moscow, Nauka Publ., 1965, 581 p. (In Russian).

Benson R.F. Harmonic auroral kilometric radiation of natural origin. Geophys. Res. Lett. 1982, vol. 9, pp. 1120-1123. DOI: 10.1029/GL009i009p01120.

Benson R.F., Calvert W. ISIS-1 observations of the source of AKR. Geophys. Res. Lett. 1979, vol. 6, p. 479.

Burinskaja T.M., Rosh Zh.L. Waveguide regime of cyclotron maser instability in plasma regions with reduced density. Fizika plazmy [Plasma Phys.]. 2007, vol. 33, no. 1, p. 28. (In Russian).

Chugunin D.V., Chernyshov A.A., Moiseenko I.L., Viktorov M.E., Mogilevskij M.M. Monitoring of the electronacceleration region with auroral kilometric radiation. Geomagnetism and Aeronomy. 2020, vol. 60, iss. 5, p. 538-546. DOI: $10.1134 /$ S0016793220040039.

Gurnett D.A. The Earth as a radio source: Terrestrial kilometric radiation. J. Geophys. Res. 1974, vol. 79, p. 4227.

Kasaba Y., Matsumoto H., Hashimoto K., Anderson R.R. The angular distribution of auroral kilometric radiation observed by GEOTAIL spacecraft. Geophys. Res. Lett. 1997, vol. 24, p. 2483.

Kasaba Y., Ishisaka K., Kasahara Y., Imachi T., Yagitani S., Kojima H., et al. Wire Probe Antenna (WPT) and Electric Field Detector (EFD) of Plasma Wave Experiment (PWE) aboard the Arise satellite: specifications and initial evaluation results. Earth, Planets and Space. 2017, vol. 69, iss. 1, article id. 174, 18 p. DOI: 10.1186/s40623-017-0760-x.

Kasahara Y., Kasaba Y., Kojima H., Yagitani S., Ishisaka K., Kumamoto A., et al. The Plasma Wave Experiment (PWE) on board the Arase (ERG) satellite. Убрать Убрать Earth, Planets and Space. 2018, vol. 70, iss. 1, article id. 86, 28 p. DOI: 10.1186/s40623-018-0842-4.

Kumamoto A., Tsuchiya F., Kasahara Y., Kasaba Y., Kojima H., Yagitani S., et al. High Frequency Analyzer (HFA) of Plasma Wave Experiment (PWE) onboard the Arase spacecraft. Earth, Planets and Space. 2018, vol. 70, iss. 1, article id. 82.14 p. DOI: 10.1186/s40623-018-0854-0.

Kurth W.S., Baumback M.M., Gurnett D.A. Direction finding measurements of auroral kilometric radiation. J. Geophys. Res. 1975 , vol. 80, p. 2764.

Louarn P., Le Quéau D. Generation of the auroral kilometric radiation in plasma cavities-II. The cyclotron maser instability in small size sources. Planet. Space Sci. 1996, vol. 44, no. 3, p. 211.

Miyoshi Y., Shinohara I., Takashima T., Asamura K., Higashio N., Mitani T., et al. Geospace Exploration Project ERG. Earth, Planets and Space. 2018a, vol. 70, iss. 1, article id. 101, 13 p. DOI: 10.1186/ s40623-018-0862-0.

Miyoshi Y., Hori T., Shoji M., Teramoto M., Chang T.F., Segawa T., et al. The ERG Science Center. Earth, Planets and Space. 2018b, vol. 70, iss. 1, article id. 96, 11 p. DOI: 10.1186/s40623-018-0867-8.
Mogilevsky M.M., Moiseenko I.L., Hanasz J. Spectral variations and long-period intensity variations of auroral kilometric radiation from INTERBALL-2 satellite measurements. Pis'ma v Astronomicheskii zhurnal [Astronomy Lett.]. 2005, vol. 31, iss. 6, pp. 422-426. DOI: 10.1134/1.1940115.

Mogilevsky M.M., Romancova T.V., Hanash Ja., Burinskaja T.M., Shrajber R. About the source of auroral kilometric radiation. Pis'ma v ZhETF [J. Experimental and Theoretical Phys. Lett.]. 2007, vol. 86, iss. 11, pp. 819-821. (In Russian).

Wu C.S., Lee L.C. A theory of the terrestrial kilometric radiation. Astrophys. J. 1979, vol. 230, p. 621.

Xiao F., Zhou Q., Su Z.,He Z., Yang C., Liu S., He Y., Gao Z. Explaining occurrences of auroral kilometric radiation in Van Allen radiation belts. Geophys. Res. Lett. 2016, vol. 43, pp. 11971-11978. DOI: 10.1002/2016GL71728.

Zarka P. Auroral radio emissions at the outer planets: Observations and theories. J. Geophys. Res. 1998, vol. 103, p. 20159. 2020).

URL: https://ergsc.isee.nagoya-u.ac.jp (accessed June 18,

URL: https://ergsc.isee.nagoya-u.ac.jp/cef/test/erg_test. cgi? site (accessed June 18, 2020).

How to cite this article

Kolpak V.I., Mogilevsky M.M., Chugunin D.V., Chernyshov A.A., Moiseenko I.L., Kumamoto A., Tsuchiya F., Kasahara Y., Shoji M., Miyoshi Y., Shinohara I. Statistical properties of auroral kilometer radiation: based on ERG (Arase) satellite data). Solar-Terrestrial Physics. 2021. Vol. 7. Iss. 1. P. 11-16. DOI: 10.12737/stp-71202102. 